\title{
A WIDE RANGE ROBUST PSS DESIGN BASED ON POWER SYSTEM POLE-PLACEMENT USING LINEAR MATRIX INEQUALITY
}

\author{
Mohammad Ataei - Rahmat-Allah Hooshmand \\ - Moein Parastegari *
}

\begin{abstract}
In this paper, a new method for robust PSS design based on the power system pole placement is presented. In this stabilizer, a feedback gain matrix is used as a controller. The controller design is proposed by formulating the problem of robust stability in a Linear Matrix Inequality (LMI) form. Then, the feedback gain matrix is designed based on the desired region of the closed loop system poles. This stabilizer shifts the poles of the power system in different operational points into the desired regions in $s$-plane, such that the response of the power system will have proper damping ratio in all the operational points. The uncertainties of the power system parameters are also considered in this robust technique. Finally, in order to show the advantages of the proposed method in comparison with conventional PSS, some simulation results are provided for a power system case study in different operational points.
\end{abstract}

K e y w o r d s: power system stabilizer (PSS), robust stability, linear matrix inequality (LMI) problem, pole placement

\section{INTRODUCTION}

The dynamic stability is one of the most important problems in the power system which can be studied by using nonlinear model of the power system. The nonlinear model of the system includes a series of nonlinear complicated equations. These nonlinear equations can be linearized around each operating point, such that small signal model of the power system can be determined. The Hefferon-Philips model is one of these power system linear models [1-19]. The studies on linear system show that the system response has not enough damping ratio and it might be unstable in some operating points [1-8]. In order to increase the damping of the power system response, classical controllers can be used which are usually designed for an operating point. If a phase lead transfer function is used in control mechanism, a conventional PSS is achieved [1-19]. Since the PSS is designed for one operational point, it has good performance only in the designed operating condition and it should be redesigned to achieve good performance in the other operational points. Therefore, the desired PSS should stabilize the power system in all operational points; $i e$, it should be robust against the changes in the operational conditions.

Adaptive control [3] and robust control strategies [415] are two main methods for solving this problem in power system which have been led to adaptive PSS and robust PSS designs respectively. Since in adaptive PSS, an identification mechanism is required to adapt the PSS parameters, the complexity of online controller computations will increase [3]. In contrast, in the robust PSS, a fixed controller for different operational points is designed [4-15] which makes it more convenient than adaptive PSS.

In the field of robust PSS, different methods based on the $H^{\infty}$ control theory [5-15] and the Quantitative Feedback Theory (QFT) [4] can be used to design a robust stabilizer, however, the QFT based robust PSS is less used because of difficulties due to its trial and error nature [4]. On the other hand, $H^{\infty}$ based methods mostly guarantee the system stability and dont ensure any other constraint on system response [9-14]. Therefore, $H^{\infty}$ method can be combined with other methods to improve performance of the PSS [5-8]. Some of these desirable methods are combination of $H^{\infty}$ and $H_{2}[5,19]$ or combination of $H^{\infty}$ and Pole-Placement methods [67]. In these combined methods, the design problem can be converted to a LMI problem, whose solving determines the stabilizer parameters [4-19].

One important issue in robust PSS design is the achievement of wide range of stability. However, this matter has not been considered in some previous studies $[8,10,16,17]$. Moreover, unlike the methods presented in references [12,18], simplified model cannot be used for power system simulations. In order to achieve desirable power system response, closed loop poles should be placed in specific zone. Although in references $[5,19]$ combined $H^{\infty}$ and $H_{2}$ methods are used, but this zonal constraint is not considered. Therefore, an important goal of this paper is to consider the above mentioned characteristics simultaneously.

On the other hand, for designing robust PSS, usually a robust strategy is used to determine lead transfer function

* Department of Electrical Engineering, Faculty of Engineering, University of Isfahan, Isfahan, Iran Hezar-Jerib St., P. Code: 8174673441, Isfahan, Iran, ataei@eng.ui.ac.ir, Hooshmand_r@yahoo.com 


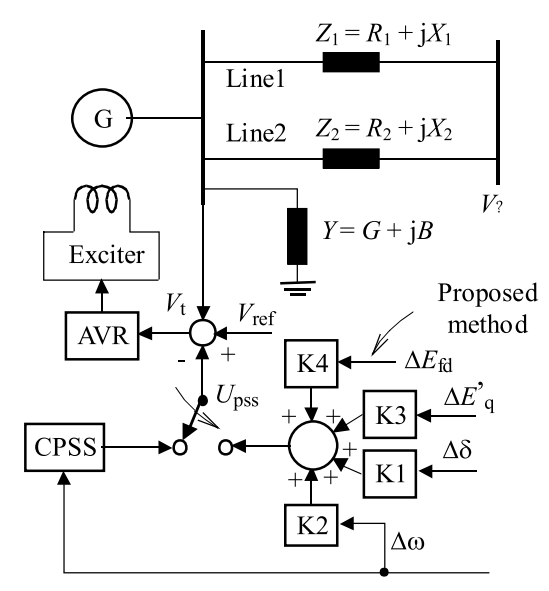

Fig. 1. Single machine infinite bus system

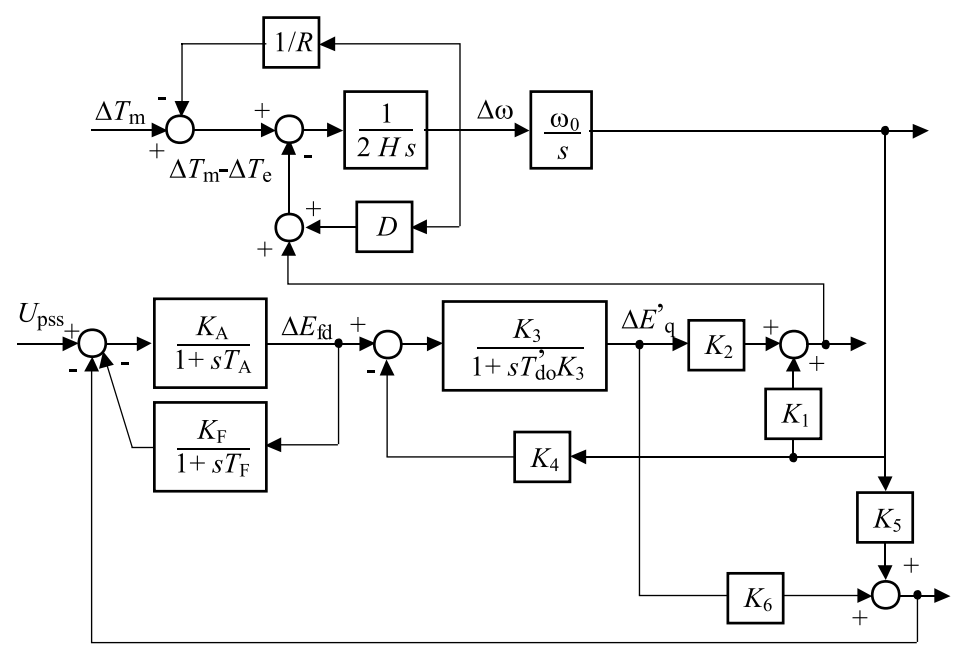

Fig. 2. The linearized model of the synchronous generator in operating point parameters [5, 13], or feedback gains [7]. If a lead transfer function is used as a controller, long settling time of power system response is achieved which is not desirable. In this regard in references $[5,13]$, although the combined $H^{\infty}$ and pole placement methods has been used to achieve wide range performance by solving LMI problem, but they use lead transfer function which has caused long transient response time.

Considering the above mentioned constraints, in this paper desig of a robust PSS based on the power system pole placement is proposed in which a feedback gain matrix is used as a controller. For this purpose, combined robust and pole placement problem is formulated as a LMI problem whose by solving, the controller parameters are determined. Also in this method, parameter uncertainty is concluded in matrix elements by considering huge number of operational points. In addition, this problem is solved such that wide range stability and short settling time is achieved simultaneously. Moreover, it can be seen that power system remains stable in emergency condition, i.e. in the operational points which is not considered in range of usual operating points. In order to show the advantages of the proposed method, some simulation results are provided for a power system case study in different operational points. It should be noted that for solving the obtained LMI problem, the MATLAB package is used and there are some numerical methods to solve the LMI problem by MATLAB [21].

The remainder of this paper is organized as follows. In Section 2, the model of the power system with uncertainty is studied. The LMI problem and its usage in robust control design are reviewed in Section 3. In Section 4 by combining robust power system stabilizer with pole placement technique, an LMI problem is obtained. The results of applying the proposed approach to a power system is reported and analyzed in Section 5. Finally, some remarkable properties of the proposed method and concluding remarks are explained in the last section.

\section{THE POWER SYSTEM MODEL WITH UNCERTAINTY}

The power system is a nonlinear system; therefore to study the small signal stability, the equations around each operating point can be linearized. On the other hand, when a single generator is connected to the infinite bus, active power $(P)$, reactive power $(Q)$ and transmission line impedance between generator and infinite bus $\left(X_{\mathrm{e}}\right)$ determine power system operating point. The power system linearized model can be used to determine the system eigenvalues. Fig. 1 shows a single line schematic diagram of a single machine infinite bus system. The generator is fitted with an Automatic Voltage Regulator (AVR) and a static excitation system. Neglecting the stator transients and the effect of damper windings, the generator and exciter can be modeled as a 4 th order system. This power system with regulator and exciter linearized model is named Hefferon Philips model which is shown in Fig. 2. In this figure, values of the $K_{1}$ to $K_{6}, K_{A}, T_{A}$ and $T_{d o}^{\prime}$ should be determined. The values of $K_{A}, T_{A}$ and $T_{d o}^{\prime}$ are the synchronous generator parameters, which are constant for each synchronous generator. On the other hand, changes in the operating point (active power $(P)$, reactive power $(Q)$ and transmission line impedance between generator and infinite bus $\left(X_{e}\right)$ ) changes $K_{1}$ to $K_{6}$ values. This model is a 4 th order model, so it has 4 state variables. The state space equations of this model are shown in the following equation.

$$
\begin{aligned}
\dot{x}(t) & =A x(t)+B u(t), \\
y & =C x(t) .
\end{aligned}
$$

where $X(t)=\left[\begin{array}{llll}\Delta \delta(t) & \Delta \omega(t) & \Delta E_{q}^{\prime}(t) & \Delta V_{F}(t)\end{array}\right]^{\top}$ is the state vector, $y$ is the output signal, $\Delta \omega(t)$ is selected as the system output, and $A$ is the state space 


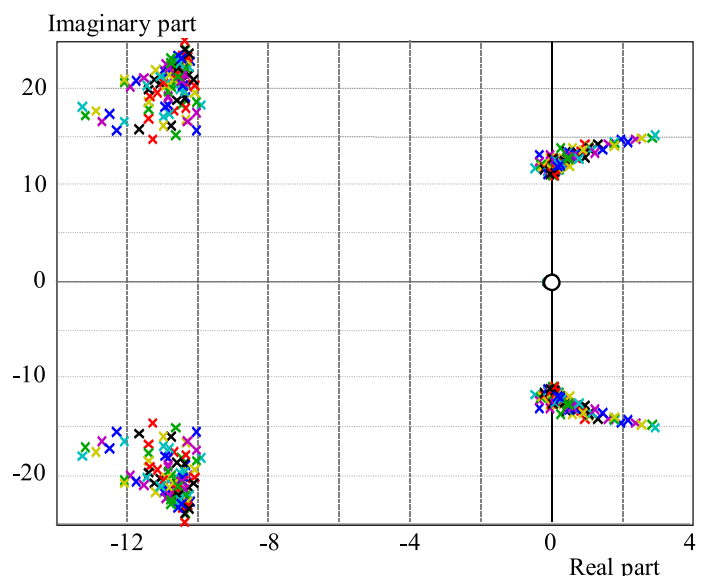

Fig. 3. Open loop poles (single machine system)

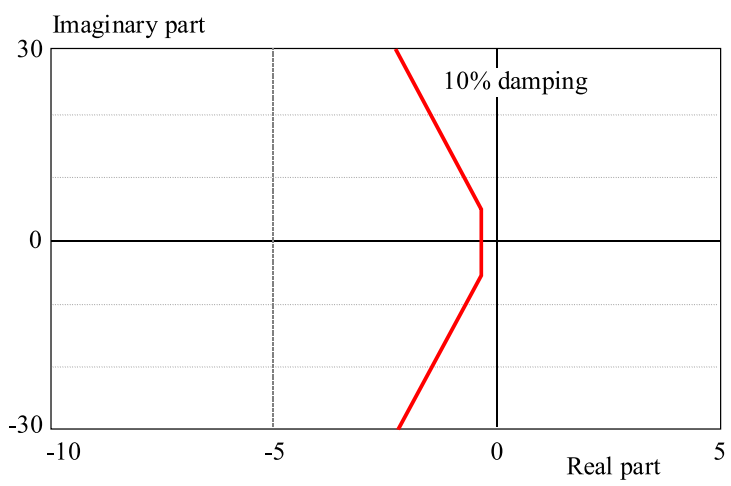

Fig. 4. $D$ shape region for pole placemen

matrix where its elements are function of the loading conditions, $B$ and $C$ are

$$
\begin{aligned}
A & =\left[\begin{array}{cccc}
0.0 & 2 \pi f & 0.0 & 0.0 \\
-\frac{K_{1}}{M} & -\frac{D}{M} & -\frac{K_{2}}{M} & 0.0 \\
-\frac{K_{4}}{T_{\mathrm{do}}^{\prime}} & 0.0 & -\frac{1}{K_{1} T_{\mathrm{do}}^{\prime}} & \frac{1}{T_{\mathrm{do}}^{\prime}} \\
-\frac{K_{\mathrm{A}} K_{5}}{T_{\mathrm{A}}} & 0.0 & -\frac{K_{A} K_{6}}{T_{\mathrm{A}}} & -\frac{1}{T_{\mathrm{A}}}
\end{array}\right], \\
B^{\top} & =\left[\begin{array}{llll}
0.0 & 0.0 & 0.0 & \frac{K_{\mathrm{A}}}{T_{\mathrm{A}}}
\end{array}\right], \\
C & =\left[\begin{array}{llll}
0.0 & 1 & 0.0 & 0.0
\end{array}\right] .
\end{aligned}
$$

The under study system specifications are provided in Appendix A. The operating condition for this system is completely defined by the values of the real power, $P$, the reactive power, $Q$, at the generator terminals and the transmission line impedance, $X_{e}$. Values of $P, Q$ and $X_{e}$, are assumed to vary independently over the following ranges: $0.2<X_{e}<0.7,-0.2<Q<0.5, \quad 0.4<P<$ 1. Changes in the operational point $\left(P, Q\right.$ and $\left.X_{e}\right)$ leads to changes in the elements of the $A$ matrix and it leads to changes in the linearized model eigenvalues. Fig. 3 shows the open loop poles for this family of plants with $P$, $Q$ and $X_{e}$, varied over the specified range by steps of 0.05 . This step distance makes a set of operational points with 1900 different members, which contains most of the operating conditions of the power system. Most of these operational points don't have adequate damping. So it is required to design a stabilizer which places the rotor mode eigenvalues inside the acceptable region as is shown in Fig. 4.

\section{LMI BASED ROBUST PSS DESIGN}

In this section, after introducing some background materials, describing the overall $H_{\infty}$ based state feedback design with pole placement in terms of LMI problem [22] which is used in the proposed method in Section 4 is presented.

\subsection{Background Materials}

This subsection discusses an LMI-based characterization for a wide class of pole clustering regions as well as an extended Lyapunov theorem for such regions. An interesting region for control purposes can be described by the set $S(\alpha, r, \theta)$ of complex numbers $x+j y$ such that

$$
x<-\alpha<0, \quad|x+j y|<r, \quad x \tan \theta<-|y| .
$$

Locating the closed-loop poles of the system in this region ensures desired performance. Let $D$ be a sub-region of the complex left-half plane. A dynamical system $\dot{x}=A x$ is called $D$-stable if all eigenvalues of the matrix $A$ lie in $D$.When $D$ is the entire left-half plane, this notion reduces to asymptotic stability, which is characterized in LMI terms by the Lyapunov theorem, ie $A$ is stable if and only if there exists a symmetric matrix $X$ satisfying

$$
A X+X A^{\top}<0, \quad X>0
$$

This Lyapunov characterization of stability has been extended to a variety of regions [22]. These regions are polynomial regions of the following form

$$
D=\left\{z \in C: \sum_{0 \leq k, l \leq m} c_{k l} z^{k} z^{-l}<0\right\}
$$

where the coefficients $c_{k l}$ are real and satisfy $c_{k l}=c_{l k}$. For polynomial regions, matrix $A$ is $D$-stable if and only if there exists a symmetric matrix $X$ such that

$$
\sum_{k, l} c_{k l} A^{k} X\left(A^{\top}\right)^{l}<0, \quad X>0 .
$$

To have the ability of synthesizing the problem in the LMI framework, it is necessary to use conditions that are affine in the state matrix $A$, such as the Lyapunov stability condition (7). Moreover, defining the LMI regions as follows is suitable for LMI-based synthesis. Hereafter, $\otimes$ denotes the Kronecker product of matrices, and the notation $M=\left[\mu_{k l}\right]_{1 \leq k, l \leq m}$ means that $M$ is an $m \times m$ matrix (block matrix) with generic entry (block) $\mu_{k l}$. 
Definition. A subset $D$ of the complex plane is called an LMI region if there exist a symmetric matrix $\alpha \in$ $\left[\alpha_{k l}\right] \in \Re^{m \times m}$ and a matrix $\beta \in\left[\beta_{k l} \in \Re^{m \times m}\right.$ such that

$$
\begin{gathered}
D=\left\{z \in C: f_{D}(z)<0\right\} . \\
f_{D}(z):=\alpha+z \beta+\bar{z} \beta^{\top}=\left[\alpha_{k l}+\beta_{k l} z+\beta_{k l} \bar{z}\right] .
\end{gathered}
$$

The pole location in a given LMI region can be characterized in terms of the following $m \times m$ block matrix

$$
\begin{aligned}
M_{D}(A, X):= & \alpha \otimes X+\beta \otimes(A X)+\beta^{\top}(A X)^{\top}= \\
& {\left[\alpha_{k l} X+\beta_{k l} A X+\beta_{l k} X A^{\top}\right]_{1 \leq k, l \leq m} . }
\end{aligned}
$$

Theorem. The matrix $A$ is $D$-stable if and only if there exists a symmetric matrix $X$ such that

$$
M_{D}(A, X)<0, \quad X>0 .
$$

Now, consider the region $S(r, \alpha, \theta)$ as defined in (8) with $\alpha=r=0$. The eigenvalues of $A$ lie in the sector $S(0,0, \theta)$ if and only if there exists a positive definite matrix $P$ such that

$$
(W \otimes A) P+P(W \otimes A)^{\top}<0
$$

where $W=\left(\begin{array}{cc}\sin \theta & \cos \theta \\ -\cos \theta & \sin \theta\end{array}\right)$. On the other hand, $S(0,0, \theta)$ is an LMI region with characteristic function

$$
f_{\theta}(z)=\left(\begin{array}{cc}
\sin \theta(z+\bar{z}) & \cos \theta(z-\bar{z}) \\
-\cos \theta(\bar{z}-z) & \sin \theta(z+\bar{z})
\end{array}\right) .
$$

$A$ has its poles in $S(0,0, \theta)$ if and only if there exists $X>0$ such that

$$
\left(\begin{array}{cc}
\sin \theta\left(A X+A X^{\top}\right) & \cos \theta\left(A X-A X^{\top}\right) \\
\cos \theta\left(X A^{\top}-A X\right) & \sin \theta\left(A X+A X^{\top}\right)
\end{array}\right)<0
$$

or equivalently

$$
(W \otimes A) \operatorname{Diag}(X, X)+\operatorname{Diag}(X, X)(W \otimes A)^{\top}<0 .
$$

In comparison with relation (14), this last condition gives additional information on the structure of $P$. It is also more suitable since the number of optimization variables is divided by four when replacing $P$ by $\operatorname{Diag}(X, X)$.

\section{2 $H_{\infty}$ Based State-Feedback Design with Pole Placement}

This subsection discusses state-feedback synthesis with $H_{\infty}$ performance and pole assignment specifications. Here, the closed-loop poles are required to lie in some LMI region $D$ contained in the left-half plane. Results are first derived in the nominal case and then extended to uncertain systems.
Consider a linear time-invariant (LTI) system described by

$$
\begin{aligned}
\dot{x}(t) & =A x(t)+B_{1} \omega(t), \\
z_{\infty}(t) & =C_{\infty} x(t)+D_{\infty 1} \omega(t)
\end{aligned}
$$

and let $T_{\omega} z_{\infty}(s)$ denote the closed-loop transfer function from $\omega$ to $z_{\infty}$ under state-feedback control $u=k x$. The constrained $H_{\infty}$ problem is to find a state-feedback gain $K$ that:

- places the closed-loop poles in some LMI stability region $D$ with characteristic function (11);

- guarantees the $H_{\infty}$ performance $\left\|T_{\omega z_{\infty}}\right\|_{\infty}<\gamma$.

Let $\left(A_{c l}, B_{c l}, C_{c l \infty}, D_{c l \infty}\right)$ denote realizations of $T_{\omega z_{\infty}}$. From mentioned theorem in Section 3.1 the pole-placement constraint is satisfied if and only if there exists $X_{D}>0$ such that

$$
\left[\alpha_{k l} X_{D}+\beta_{k l} A_{c l} X_{D}+\beta_{l k} X_{D} A_{c l}^{\top}\right]_{1 \leq k, l \leq m}<0 .
$$

Meanwhile, the $H_{\infty}$ constraint is equivalent to the existence of a solution $X_{\infty}>0$ for the following LMI:

$$
\left(\begin{array}{ccc}
A_{c l} X_{\infty} & B_{c l} & X_{\infty} C_{c l \infty}^{\top} \\
B_{c l}^{\top} & -I & D_{c l \infty}^{\top} \\
C_{c l \infty} X_{\infty} & D_{c l \infty} & -\gamma^{2} I
\end{array}\right)<0 .
$$

This result is known as the Bounded Real Lemma.

Our goal is to determine state feedback gains $K$ that satisfies the $H_{\infty}$ and pole constraints. From the previous discussion, this is equivalent to satisfy (19), (20). While this problem is not jointly convex in the variables $\left(X_{D}, X_{\infty}, Y, K\right)$, convexity can be enforced by finding a common solution

$$
X=X_{D}=X_{\infty}>0 .
$$

Consequently LMI constraints can be summarized as the following inequalities

$$
\begin{gathered}
{\left[\alpha_{k l} X+\beta_{k l} U(X, L)+\beta_{l k} U(X, L)^{\top}\right]<0,} \\
\left(\begin{array}{ccc}
U(X, L)+U(X, L)^{\top} & B_{1} & V(X, L)^{\top} \\
B_{1}^{\top} & -I & D_{\infty 1}^{\top} \\
V(X, L) & D_{\infty 1} & -\gamma^{2} I
\end{array}\right)<0 \\
U(X, L):=A X, \quad V(X, L):=C_{\infty} X
\end{gathered}
$$

In this stage, LMI optimization software such as the MATLAB LMI Control Toolbox can be used to solve the problem. 


\section{THE PROPOSED WIDE RANGE ROBUST PSS BY USING LMI}

\subsection{Design Objectives}

The Power system eigenvalues which show the relative stability of the system are determined by its linearized model. These eigenvalues have not enough damping to stabilize power system response. It should be noted that in power systems, a damping factor, $\zeta$, of at least $10 \%$ and a real part, $\sigma$, not greater than -0.5 for the troublesome low frequency electromechanical mode, guarantees that the excited low frequency oscillations will damp down in a reasonably short time. Such restriction on all the system eigenvalues would imply that all the poles of the system lie in the left of the imaginary axis in a $D$ shape contour. This $D$ shape contour is shown in Fig. 4. If a power system has above specifications, low frequency oscillations will damp after a short time. However, a real power system has not such a good structure and all of its poles have not enough damping and might be unstable. To stabilize the power system response, feedback controllers are used. The feedback gains should be determined such that the closed loop eigenvalues shift to the $D$ shape region. These feedback gains should also be small enough to prevent controllers saturation.

On the other hand, in this paper our goal is finding the feedback gains such that it guarantees stable power system response in spite of changes in operational point. A robust controller and a well damped response is obtained in a wide range of operating points, if proposed method guarantees acceptable small signal transient in all operational points.

For this purpose, the robust controller design problem is converted to a LMI problem. Thus, solving the LMI problem leads to robust power system stabilizer. To accomplish this, at first, system uncertainties should be studied in the state space equations.

\subsection{Representing the uncertainty in the power system model}

Dynamical behavior of a system can be described by state space equations. These state space equations consist of power system parameters which are not constant. Therefore, the system should be determined by an uncertain state space model.

The state space equations of the uncertain system can be considered as follows

$$
\begin{aligned}
E \dot{x} & =A x(t)+B u(t), \\
y(t) & =C x(t)+D u(t) .
\end{aligned}
$$

where the matrices $A, B, C, D, E$ depend on uncertain parameters which vary in some bounded sets. The matrix elements boundaries can be determined in $\mathrm{n}$ operating points. Therefore changes in the operating point leads to changes in the state space equations.
In the resultant, considering the uncertain elements of each uncertain matrix, the matrices $A$ and $B$ can be considered as the following form

$$
(A, B) \in\left\{\left(\sum_{i=1}^{N} p_{i} A_{i}, \sum_{i=1}^{N} p_{i} B_{i}\right): \sum_{i=1}^{N} p_{i}=1, p_{i} \geq 0\right\} .
$$

Such polytopic models may be resulted from convex interpolation of a set of models $(A, B)$ identified in different operating points. They also arise in connection with affine parameter dependent models as

$$
\dot{x}=A(p) x+B(p) u .
$$

where $p$ is a vector of real uncertain parameters and $A(p), B(p)$ are affine matrix-valued functions of $p$.

The uncertain matrix $A$ of the under study power system based on the presented model in Section 2 is as follows

$$
A=\left[\begin{array}{cccc}
0.0 & a_{12} & 0.0 & 0.0 \\
a_{21} & a_{22} & a_{23} & 0.0 \\
a_{31} & 0.0 & a_{33} & a_{34} \\
a_{41} & 0.0 & a_{43} & a_{44}
\end{array}\right],
$$

The system matrix $A(k)$ is affinely dependent on $k$, ie $A(k)$ can be rewritten in the following form

$$
A_{0}+a_{21} A_{1}+a_{23} A_{2}+a_{31} A_{3}+a_{33} A_{4}+a_{41} A_{5}+a_{43} A_{6}
$$

where $A_{0}, A_{1}, A_{2}, A_{3}, A_{5}$ and $A_{6}$ are constant matrices. Under different loading conditions each parameter varies within a certain range as:

$a_{21} \in\left[a_{21}^{-}, a_{21}^{+}\right], \quad a_{23} \in\left[a_{23}^{-}, a_{23}^{+}\right], \quad a_{31} \in\left[a_{31}^{-}, a_{31}^{+}\right]$, $a_{33} \in\left[a_{33}^{-}, a_{33}^{+}\right], a_{41} \in\left[a_{41}^{-}, a_{41}^{+}\right]$and $a_{43} \in\left[a_{43}^{-}, a_{43}^{+}\right]$ where $a_{i j}^{-}$and $a_{i j}^{+}$denotes the lower and upper bound of the parameter $a_{i j}$ respectively for all $P \in\left[P^{-}, P^{+}\right]$, $Q \in\left[Q^{-}, Q^{+}\right]$and $x_{e} \in\left[x_{e}^{-}, x_{e}^{+}\right]$. These bounds can be calculated using any standard optimization technique. This affine parameter-dependent model can be converted to a polytopic model. The parameter vector $\left[\begin{array}{llllll}a_{21} & a_{23} & a_{31} & a_{33} & a_{41} & a_{43}\end{array}\right] \in \Re^{6}$ takes values in a parameter-box with $2^{5}=32$ corners

$$
\begin{aligned}
k_{\text {cor } 1} & =\left[\begin{array}{llllll}
a_{21}^{-} & a_{23}^{+} & a_{31}^{+} & a_{33}^{+} & a_{41}^{+} & a_{43}^{+}
\end{array}\right]^{\top}, \\
k_{\text {cor } 2} & =\left[\begin{array}{llllll}
a_{21}^{+} & a_{23}^{-} & a_{31}^{+} & a_{33}^{+} & a_{41}^{+} & a_{43}^{+}
\end{array}\right]^{\top}, \\
& \vdots \\
k_{\text {cor } 32} & =\left[\begin{array}{llllll}
a_{21}^{-} & a_{23}^{-} & a_{31}^{-} & a_{33}^{-} & a_{41}^{-} & a_{43}^{-}
\end{array}\right]^{\top} .
\end{aligned}
$$

Since $A(k)$ is affine in $k$, it maps this parameter box to a polytope of matrices with 32 vertices defined at each parameter box corner. 


\subsection{Design Procedure}

By considering above uncertainty description, feedback gains should be determined such that the power system eigenvalues places in the desirable $D$ region. It should be noted that an unnecessarily large shift of the system poles into the left half plane should be avoided, since this may lead to large feedback gains. Therefore, imposition of constraints enforces the closed loop poles to a $\mathrm{D}$ shape region specified with $\theta<45^{\circ}$ and $-50 \leq$ $\sigma \leq-0.5$. In the other word, the open loop poles of the whole family of plants should be shifted to this region.

In Section 3 the issue of converting the state feedback design to an LMI problem was discussed. These results can be extended for uncertain systems [22]. For brevity, in the following only converting the pole placement problem by considering the uncertainty description of Subsection 4.2 is discussed. Thus, solving the resultant LMI problem leads to design of feedback gains, which is robust against changes in the $P, Q$ and $X_{e}$. Consequently, the power system eigenvalues are forced into the $D$ shape region in the left half plane.

Consider the problem of computing a state feedback gain $K$ that forces the closed-loop eigenvalues into some LMI region $D$ for all admissible values of $A$ and $B$. The equation (25) is called quadratically $D$-stabilizable if there exists a gain $K$ and a single Lyapunov matrix $X>0$ such that $M_{D}(A+B K, X)<0$ for all admissible values of $A$ and $B$. In the continue, it is shown that this condition is also necessary, and the results are extended to arbitrary LMI regions.

Let $D$ be any LMI region, suppose that (25) is quadratically $D$-stabilizable with Lyapunov matrix $X$ and state-feedback gain $K$, and let $L:=K X$. By considering the condition $M_{D}(A+B K, X)<0$ at each vertex $\left(A_{i}, B_{i}\right)$ of $(27)$, the following necessary conditions on $X$, $L$ are achieved

$$
\begin{array}{r}
{\left[\alpha_{k l} X+\beta_{k l}\left(A_{i} X+B_{i} L\right)+\beta_{l k}\left(A_{i} X+B_{i} L\right)^{\top} T\right]_{k, l}<0} \\
\text { for } i=1, \ldots, N, \quad
\end{array}
$$

$$
X>0 .
$$

Conversely, it can be seen that any solution $(L, X)$ of this LMI system satisfies $M_{D}(A+B K, X)<0$ when forming the weighted sum of the LMIs (32) with nonnegative coefficients $p_{1}, \ldots, p_{N}$. Hence, the relations (32) and (33) are necessary and sufficient for quadratic $D$-stabilizability. Note that LMI conditions for quadratic $H_{\infty}$ performance over (27) are obtained similarly by writing (22), (23) at each vertex of the polytopic plant.

Now, it is proposed to apply a full state feedback controller using the described LMI based approach for achieving the robust D-stability requirement. Each state is measured, multiplied by the appropriate gain and then summed up before being fed at the reference input of the Automatic Voltage Regulator. In a practical implementation, additional hardware would be required for state measurements. For the 4 th order model used here, the states are the deviations in the load angle $\delta$, rotor speed $\omega$, field voltage $E_{f d}$ and the internal voltage $E_{q}^{\prime}$. The values of $\delta, \omega$ and $E_{f d}$ can be directly measured using appropriate transducers. The internal voltage $E_{q}^{\prime}$ can be computed from the instantaneous values of the stator currents and the equivalent circuit parameters. A polytopic system is obtained for this set by choosing the $A$ and $B$ matrices corresponding to the external values of $P, Q$ and $X_{e}$ as stated in Subsection 4.2. All possible combinations of the minimum and maximum values of each of these 3 parameters are taken to generate a set of 8 vertex systems corresponding to the 8 corners of a cube in the space with $P, Q$ and $X_{e}$ coordinates. Since the point with minimum $Q$ and maximum $P$ and $X_{e}$, did not have a steady state load flow solution, it was replaced by a nearby feasible point as $P=1.0, Q=-0.2$, $X=0.45$.

\section{SIMULATION RESULTS}

\subsection{Under study system}

In order to investigate the performance of the proposed method, it is used to stabilize low frequency oscillations of a generator connected to the infinite bus which is shown in Fig. 1. The Power system linearized model, Hefferon-Philips model, is shown in Fig. 2. The parameters of the under study synchronous generator connected to infinite bus are provided in Appendix A. To examine the robustness of the proposed method, this system is studied in three different operating conditions. These operating points are specified as follow:

First operating point.

$$
\begin{aligned}
Q & =0.55, P=0.8, X_{e}=0.4, \\
& \Longrightarrow\left\{\begin{array}{l}
K_{1}=0.97, K_{2}=0.97, K_{3}=0.36, \\
K_{4}=1.24, K_{5}=-0.05, K_{6}=0.46
\end{array}\right.
\end{aligned}
$$

Second operating point.

$$
\begin{aligned}
& p f=0.82, P=1.2, X_{e}=0.6, \\
& \Longrightarrow\left\{\begin{array}{l}
K_{1}=1.75, K_{2}=1.1145, K_{3}=0.4182, \\
K_{4}=1.42, K_{5}-0.19, K_{6}=0.5459 .
\end{array}\right.
\end{aligned}
$$

Third operating point.

$$
\begin{aligned}
& Q=0.5, P=1, X_{e}=0.7 \\
& \Longrightarrow\left\{\begin{array}{l}
K_{1}=0.97, K_{2}=0.96, K_{3}=0.42, \\
K_{4}=1.228, K_{5}=-0.12, K_{6}=0.536 .
\end{array}\right.
\end{aligned}
$$

By considering $Q=0.55 \mathrm{pu}$ in (34) and $P=1.2 \mathrm{pu}$ in (35), it is seen that the first and second operating points are even out of the predefined operating ranges $0.2<X_{e}<0.7,-0.2<Q<0.5,0.4<P<1$.

Also, by considering (36), it is concluded that the third operating point is at the corner of the conventional operating conditions. 


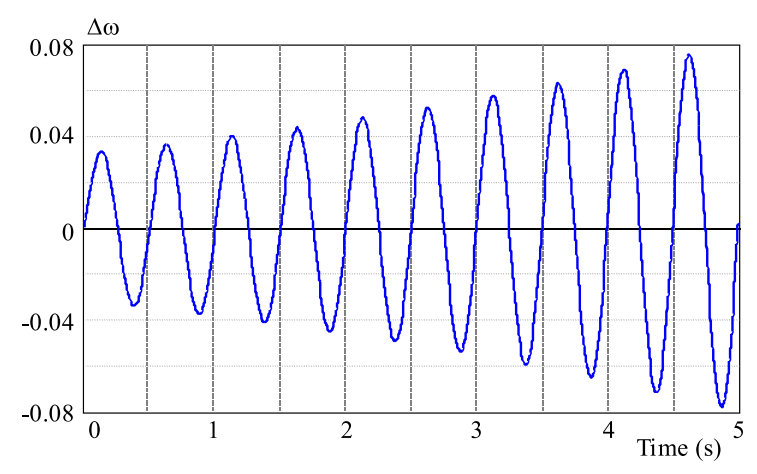

Fig. 5. The response of $\Delta \omega(t)$ with respect to time in the first operating point without using stabilizer

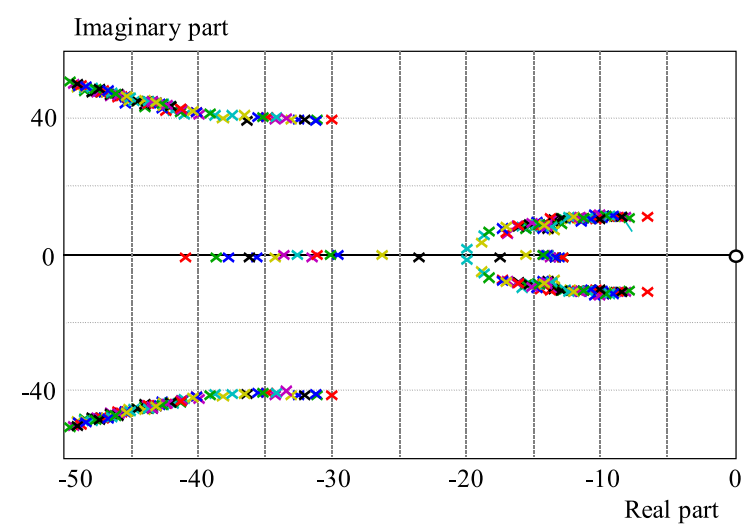

Fig. 7. Closed loop poles with the proposed state feedback controller for $D$ shape region pole placement

The performance of the proposed method in three different operating points is compared with conventional PSS to illustrate the proposed method robustness and desirable dynamic performance. For this purpose, open loop responses of the power system $(\Delta \omega(t))$ for these operating points are shown in Figs. 5 and 6 .

\subsection{Designing robust stabilizer with pole place- ment by Solving LMI Problem}

By applying the proposed method in Section 4, the feedback matrix gain is achieved for the under study power system

$k=\left[\begin{array}{ll}-0.5899466391 & 292.6014667524\end{array}\right.$

$$
-9.37859374-0.0407642254] \text {. }
$$

Figure 7 shows the closed loop poles of the set of plants with the stipulated variations in $P, Q$ and $X_{e}$. As seen, the eigenvalues has been shifted into the desired region of the complex plane for the entire set of plants. Also Fig. 8 shows closed loop eigenvalues if feedback gains determination problem is solved for oval shaped LMI.

\subsection{Designing Conventional PSS}

Power system stabilizers can extend power transfer stability limits which are characterized by lightly damped

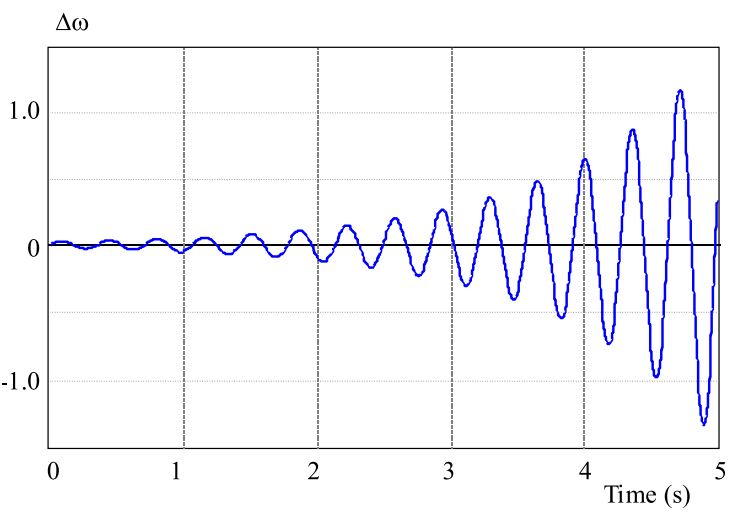

Fig. 6. The response of $\Delta \omega(t)$ with respect to time in the second operating point without using stabilizer

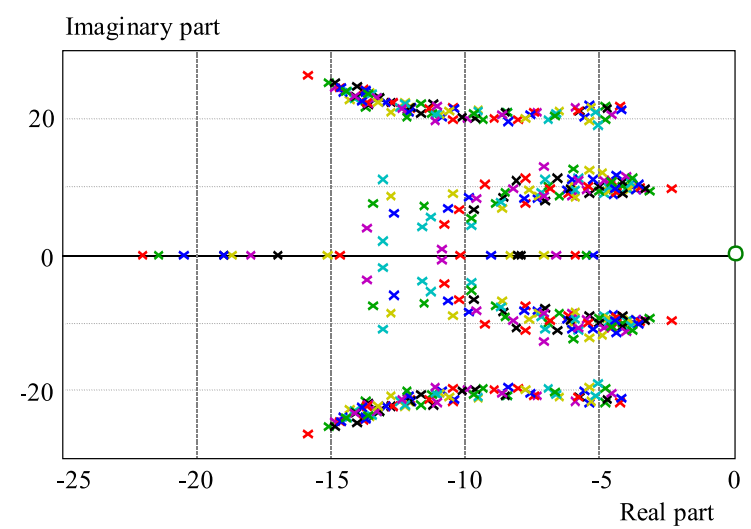

Fig. 8. Closed loop poles with the proposed state feedback controller for oval region pole placement

or spontaneously growing oscillations in the 0.2 to $2.5 \mathrm{~Hz}$ frequency range. This is accomplished via excitation control, providing damping to the systems oscillation modes. Consequently, the important issue is the stabilizers ability to enhance damping under the least stable conditions, $i e$ the performance conditions.

Conventional PSS is a controller which stabilize a power system and has a lead-lag transfer function and damp response of the low frequency oscillations $(\Delta \omega)$. A high pass filter is used before lead-lag controller to prevention harmful effects of the dc signal or very low frequency changes. Finally PSS transfer function obtained like $H_{P S S}(s)=\frac{K_{C} s}{1+T_{w} s} \frac{T_{1}+s}{T_{2}+s}$ form. In this stabilizer $T_{1}$ and $K_{C}$ are the controller parameters. In this study, we design the conventional PSS for the second operating point.

\subsection{Comparison between proposed stabilizer and conventional PSS}

In order to compare the performance of the proposed stabilizer and conventional PSS, the system response $\Delta \omega(t)$ in the case of applying these stabilizers for three different operating points are provided in Figs. 9 to 11. As shown in these figures, proposed stabilizers response has less overshoot and settling time with respect to conven- 


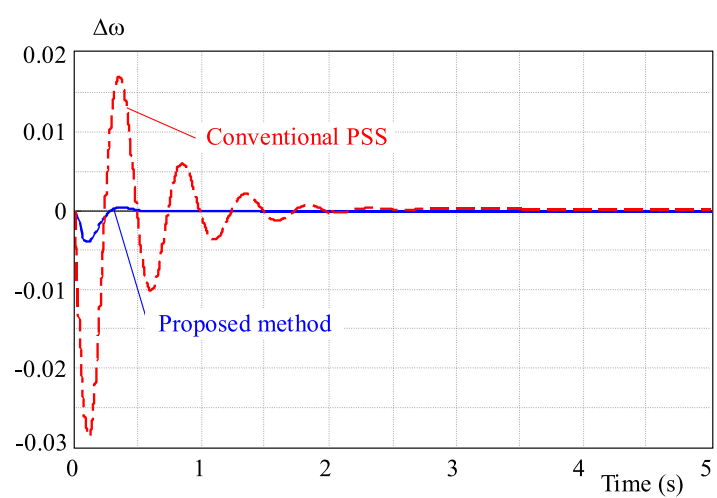

Fig. 9. The response of $\Delta \omega(t)$ with respect to time in the first operating point by using proposed robust stabilizer compared with conventional PSS

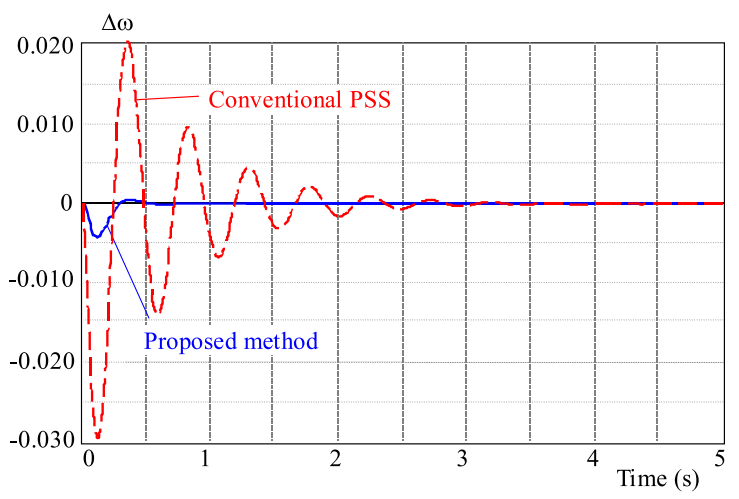

Fig. 11. The response of $\Delta \omega(t)$ with respect to time in the third operating point by using proposed robust stabilizer and conventional PSS

Table 1. Overshot and settling time of the proposed robust stabilizer's response $\Delta \omega(t)$

\begin{tabular}{c|ccc}
\hline & \multicolumn{3}{|c}{ Operating point } \\
& First & Second & Third \\
\hline Settling time & 0.405 & 0.312 & 0.33 \\
Overshoot & 0.003971 & 0.00373 & 0.003897 \\
\hline
\end{tabular}

Table 2. Overshot and settling time of the conventional stabilizer's response $\Delta \omega(t)$

\begin{tabular}{c|ccc}
\hline & \multicolumn{3}{|c}{ Operating point } \\
& First & Second & Third \\
\hline Settling time & 1.59 & 1.93 & 1.96 \\
Overshoot & 0.02903 & 0.02242 & 0.02871 \\
\hline
\end{tabular}

tional PSS. In other word, the performance of the power system is much desirable in the case of using the proposed stabilizer for all operating condition. It should be noted that the first and second operating points are even out of range for which the proposed robust stabilizer has been designed. Also, third operating point is also a boundary operating point at the corner of the operating point

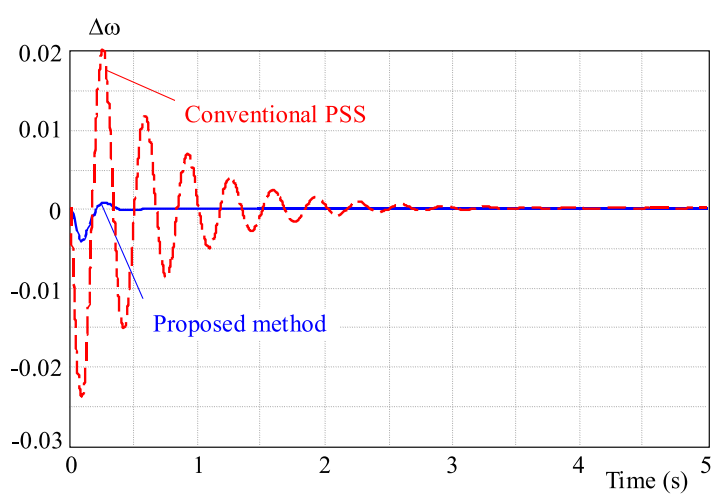

Fig. 10. The response of $\Delta \omega(t)$ with respect to time in the second operating point by using proposed robust stabilizer and conventional PSS

range. In order to compare the performance of both stabilizers quantitatively, the overshoot and settling time of the responses in all operating points have been provided in Tabs. 1 and 2 for the proposed and conventional PSS respectively. By comparing them, it is concluded that proposed stabilizer has less overshoot and settling time compare with conventional stabilizer. Furthermore, it is illustrated that proposed stabilizer, stabilize power system oscillations not only for the proposed operating point range, but also for other operating point out of the range of design problem.

\section{CONCLUSION}

In this paper, a new method for robust PSS design is presented. For this purpose, designing robust stabilizer problem by specified eigenvalues region is converted to a LMI problem; therefore solving LMI problem leads to finding robust state feedback, which is computed based on the region placement of the system poles. Consequently, this stabilizer shifts the power system poles in different operational points into the desired regions in $s$-plane, such that the response of the power system will have proper damping ratio in all the operational points. It provides the desired closed loop performance over the prespecified range of operating conditions. This stabilizer decreases overshoot and settling time of the power system response. Finally, to illustrate proposed stabilizer advantages, proposed stabilizer is compared with conventional PSS. Consequently, designing a new robust power system stabilizer based on pole placement technique is converted to a LMI problem, solving this LMI problem leads to determining feedback gains robust which makes power system robust with enough damping against changes in operational points.

\section{Appendix A - The model parameters of the case study}

The parameters of the model and the synchronous generator, which is used in this study, are given as follow. All values are in per unit (pu) except the $H$ and $T$ that are in second: $H=5 \mathrm{sec}, \quad T_{d o}^{\prime}=6 \mathrm{sec}, \quad X_{c}=1.6 \mathrm{pu}, X_{q}=$ 
$1.55 \mathrm{pu}, \quad X_{d}^{\prime}=0.32 \mathrm{pu}$ Also, the system excitation parameters are $K_{A}=400, K_{F}=0.025, T_{A}=$ $0.05 \mathrm{sec}, T_{F}=1 \mathrm{sec}$. Moreover, the parameters, which are used in the controller design, are

$$
\begin{array}{ll}
P_{1 \text {-self-tuning }}=-1.07, & P_{2 \text {-self-tuning }}=0.6376, \\
\omega_{\text {conventional-PSS }}=7.6, & \zeta_{\text {conventional-PSS }}=0.2, \\
T_{2 \text {-conventional-PSS }}=0.2, & K_{\text {conventional-PSS }}=5.622 .
\end{array}
$$

\section{REFERENCES}

[1] ANDERSON, P. M.-FOUAD, A. A.: Power System Control and Stability, IEEE Press, 2002.

[2] YU, Y. N.: Electric Power System Dynamic Stability, Academic press, 1983.

[3] Ataei, M.-HoOshmand, R.-PARASTEGari, M. : SelfTuning Power System Stabilizer Design Based on Pole Assignment and Pole-Shifting Techniques, Journal of Applied Sciences 8 No. 8 (2008), 1406-1415.

[4] WERNER, H.-KORBA, P.-YANG, T. C. : Robust Tuning of Power System Stabilizers Using LMI-Techniques, IEEE Trans. on Control Systems Technology 11 No. 1 (Jan 2003), 147-152.

[5] SOLIMAN, M.-EMARA, H.-ELSHAFEI, A.-BAHGAT, A.-MALIK, O. P.: Robust Output Feedback Power System Stabilizer Design: an LMI Approach, IEEE Power and Energy Society General Meeting - Conversion and Delivery of Electrical Energy in the $21^{\text {st }}$ Century, July 2008, pp. 1-8.

[6] Chilali, M.-GAhinet, P.: $H^{\infty}$ Design with Pole Placement Constraints: An LMI approach, IEEE Trans. on Automatic Control 41 No. 3 (Mar 1996), 358-367.

[7] RAO, P. S.-SEN, I. : Robust Pole Placement Stabilizer Design Using Linear Matrix Inequalities, IEEE Trans. Power Systems 15 No. 1 (Feb 2000), 313-319.

[8] GUPTA, R.-BHATIA, D. : Comparison of Robust Fuzzy Logic and Fast Output Sampling Feedback Based Power System Stabilizer for SMIB, IEEE International Conf. on Industrial Technology, Dec 2006, pp. 1031-1036.

[9] CHOW, J. T.-HARRIS, P.-OTHMAN, H. A.-SNNCHEAGUSCA, J. J.-TERWILLIGER, C. E. : Robust Control Design of Power System Stabilizers Using Multivariable Frequency Domain Techniques, in Proc. $29^{\text {th }}$ IEEE Conf. on Decision and Control, Hawai, Dec 1990, pp. 2067-2073.

[10] SCAVONI, F. E.- de SILVA, A. S.-NETO, A. T.-CAMPAGNOLO, J. M.: Design of Robust Power System Controllers using Linear Matrix Inequalities, Proc. 2001 IEEE Porto Power Tech Conf., vol. 2, Sep 2001, pp. 1-6.

[11] JIANYING, H. G.-RONG, X.-WEIGUO, L.-SHUANQIN, X. : H Infinity Controller Design of the Synchronous Generator, International Conference on Intelligent Computation Technology and Automation (ICICTA), vol. 1, Oct 2008, pp. 370-374.

[12] DEHGHANI, M.-NIKRAVESH, S. K. Y.: Robust Tuning of PSS Parameters Using the Linear Matrix Inequalities Approach, IEEE Power Tech 2007 Lausanne, July 2007, pp. 322-326.

[13] JOO, K. S.-MOON, K. S.-HYUN, Y.-KOOK-HUN, K.: Low-Order Robust Power System Stabilizer for Single-Machine Systems: an LMI Approach, $32^{\text {nd }}$ Annual IEEE Conf. on Industrial Electronics, IECON 2006, Nov 2006, pp. 742-747.

[14] GUPTA, R.-BANDYOPADHYAY, B.-KULKARNI, A. M. : Design of Power System Stabilizer for Single-Machine System using Robust Periodic Output Feedback Controller, Proc. 2003 IEE Generator Transmission Distribution Conf., vol. 150, Mar 2003, pp. 211-216.
[15] JIANYING, G.-RONG, X.-WEIGUO, L.-SHUANQIN, X. : $H_{\infty}$ Controller Design of the Synchronous Generator, International Conf on Intelligent Computation Technology and Automation (ICICTA), vol. 1, Oct 2008, pp. 370-374.

[16] SHIAU, J. K.-CHOW, J. H.—BOUKARIM, G.: Power Swing Damping Controller Design using Linear Matrix Inequality Algorithm, Proc. IEEE Conf Control Application, vol. 2, Sep 1996, pp. $727-732$.

[17] El-RAZAZ, Z. S.-MANDOR, M. E. D.-ALI, E. S. : Damping Controller Design for Power Systems Using LMI and GA Techniques, Proceedings of the $41^{\text {st }}$ International Universities Power Engineering Conf., vol. 2, Sep 2006, pp. 500-506.

[18] JUHUA,-KROGH, B. H.-ILIC, M. D. : Saturation-Induced Frequency Instability in Electric Power Systems, IEEE Power and Energy Society General Meeting - Conversion and Delivery of Electrical Energy in the $21^{\text {st }}$ Century, July 2008, pp. 1-7.

[19] BEVRANI, H.-HIYAMA, T.: Stability and Voltage Regulation Enhancement using an Optimal Gain Vector, Proc. IEE Power Engineering Society Conf., June 2006, pp. 1-8.

[20] BOYD, S.-FERON, E.-BALAKRISHNAN, V.-GHAOUI, E. L.: Linear Matrix Inequalities in System and Control Theory, Studies in Applied Mathematics, SIAM, Philadelphia PA, 1994.

[21] GAHINET, P.-NEMIKOVSKI, A.-LAUR, A. J.-CHILALI, M. : LMI Control Toolbox for use with Matlab, The Mathworks Inc., Natick, MA, 1995.

22] Chilali, M.-GAhinet, P.: $H_{\infty}$ Design with Pole Placement Constraints: An LMI Approach, IEEE Trans. Automatic Control 41 No. 3 (Mar 1996), 358-367.

Received 31 May 2010

Mohammad Ataei (1971) received the BS degree from the Isfahan University of Technology, Iran, in 1994, the MS degree from the Iran University of Science \& Technology, Iran, in 1997, and PhD degree from K. N. Toosi University of Technology, Iran, in 2004 all in Electrical Engineering. He has done his $\mathrm{PhD}$ project jointly with the University of Bremen in Germany from 2001 till 2003. Since 2004, he is with the Department of Electrical Engineering at the University of Isfahan, Iran. He has worked on time series analysis of chaotic systems, robust control, control theory and applications. His main areas of research interest are also chaos control and synchronization, and nonlinear control.

Rahmat-Allah Hooshmand (1967) received the BSEE degree from the University of Mashhad in 1989, the MSEE from the University of Tehran/Iran and PhD degree from Tarbiat Modarres University/Iran in 1990 and 1995, respectively all in Electrical Engineering. Since 1995, he is with the Department of Electrical Engineering at the University of Isfahan/Iran, as an associate professor. His main areas of research interest are modeling of Power Systems and Distribution Networks.

Moein Parastegari (1983) was born in Isfahan in the Islamic Republic of Iran, on March 30, 1983. He received BS Degree in Electrical Engineering from Shahrekord University, Iran, in 2005. He received MS Degree in Electrical Engineering from University of Isfahan, Iran, in 2008. Currently, he is working towards the PhD Degree in power system from Isfahan University, Isfahan, Iran. His research interests are in the area of power systems control and operation. 